\title{
Gas-phase Lifetimes of Nucleobase Analogues by Picosecond Pump- ionization and Streak Techniques
}

\author{
Susan Blaser $\$$, Hans-Martin Frey, Cornelia G. Heida, and Samuel Leutwyler ${ }^{\star}$
}

§SCS-Metrohm Foundation Award for best oral presentation

\begin{abstract}
The picosecond (ps) timescale is relevant for the investigation of many molecular dynamical processes such as fluorescence, nonradiative relaxation, intramolecular vibrational relaxation, molecular rotation and intermolecular energy transfer, to name a few. While investigations of ultrafast (femtosecond) processes of biological molecules, e.g. nucleobases and their analogues in the gas phase are available, there are few investigations on the ps time scale. We have constructed a ps pump-ionization setup and a ps streak camera fluorescence apparatus for the determination of lifetimes of supersonic jet-cooled and isolated molecules and clusters. The ps pump-ionization setup was used to determine the lifetimes of the nucleobase analogue 2-aminopurine (2AP) and of two 2AP. $\left(\mathrm{H}_{2} \mathrm{O}\right)_{n}$ water cluster isomers with $\mathrm{n}=1$ and 2 . Their lifetimes lie between $150 \mathrm{ps}$ and $3 \mathrm{~ns}$ and are strongly cluster-size dependent. The ps streak camera setup was used to determine accurate fluorescence lifetimes of the uracil analogue 2-pyridone (2PY), its self-dimer $(2 \mathrm{PY})_{2}$, two isomers of its trimer $(2 \mathrm{PY})_{3}$ and its tetramer $(2 \mathrm{PY})_{4}$, which lie in the 7-12 ns range.
\end{abstract}

Keywords: 2-Aminopurine · Lifetimes · Picosecond pump-ionization · Picosecond streak camera $\cdot$ 2-Pyridone

\section{Introduction}

Many molecular dynamic processes take place on the picosecond (ps) time scale. These include relaxation processes such as fluorescence, internal vibrational relaxation (IVR), intersystem crossing (ISC), molecular rotation, collisional energy transfer and dephasing and intermolecular electronic energy transfer. The development of stable ps sources in the early 70s allowed fluorescence and Raman decay measurements ${ }^{[1,2]}$ and first pump-probe experiments by Lauberau. ${ }^{[3]}$ Hochstrasser et al. extended the range from the visible to the infrared (IR) and performed ps experiments on photochemi-

\footnotetext{
${ }^{*}$ Correspondence: Prof. Dr. S. Leutwyler Universität Bern

Departement für Chemie und Biochemie Freiestrasse 3

$\mathrm{CH}-3012$ Bern

Tel.: +41316314479

E-mail: leutwyler@iac.unibe.ch

${ }^{a}$ Current address: Dept. of Chemistry

University of Wisconsin-Madison

Madison, Wisconsin 53706, USA
}

cal predissociation and fluorescence in the condensed phase. ${ }^{[4-6]}$ Zewail and co-workers transferred these methods into the gasphase and to molecular beams, including time-resolved structural changes by ultrafast electron diffraction techniques. ${ }^{[7-9]}$

Recent ps investigations on gas-phase molecules have focused on ground- and excited state IVR, which occurs on a timescale of 0.5-100 ps. Mikami and coworkers have studied the ground state IVR dynamics of organic UV chromophores and clusters using ps IR-UV pump-probe experiments, and for instance have determined the IVR lifetime of the $\mathrm{OH} v=1$ vibrational stretch of phenol as 14 ps. ${ }^{[10,11]}$ Reid and co-workers have investigated IVR mechanisms in electronically excited states by combining ps time-resolved photoelectron spectroscopy with the pumpionization method. ${ }^{[12]}$ This yields ps timeresolved information on the internal state distribution of the newly-formed ion and thereby on the IVR process of the excitedstate neutral molecule.[12] These selected examples show that the ps time range is of great importance in the understanding of intramolecular dynamics.

Here we describe a pulsed supersonic beam apparatus that combines two different ps techniques with a laser spectroscopic resolution of about $10 \mathrm{~cm}^{-1}$ in the UV between 215 and $350 \mathrm{~nm}$ (Fig. 1): A pump/ ionization experimental setup (A) is able to resolve excited-state lifetimes from $\sim 20$ ps up to $\sim 3$ ns. This is complemented by a ps streak-camera setup (B) for measuring decays over the $50 \mathrm{ps}$ to $100 \mathrm{~ns}$ time range. As an application of the pump-probe setup, we present lifetimes of $9 \mathrm{H}$-2-aminopurine (2AP), which is a weakly fluorescent isomer of adenine (6-aminopurine) and of its water clusters $2 \mathrm{AP} \cdot\left(\mathrm{H}_{2} \mathrm{O}\right)_{n}$ with $n=1$ and 2 . The ps streak camera setup was used to investigate the fluorescent cis-amide 2-pyridone (2PY) and its dimer, trimers and tetramer. All molecules/clusters were cooled to $\sim 3 \mathrm{~K}$ in a pulsed supersonic molecular beam.

\section{Experimental Setups}

\subsection{Picosecond Pump-Ionization Setup}

Fig. 1 shows a scheme of the ps pumpionization apparatus (A). The molecular sample is placed in a $20 \mathrm{~Hz}$ magnetically pulsed supersonic jet nozzle and heated to a vapor pressure of $0.2-2 \mathrm{mbar}$, corresponding to temperatures of $80{ }^{\circ} \mathrm{C}$ to 230 ${ }^{\circ} \mathrm{C}$. The vapor is entrained in a noble gas carrier at 1-2 bar (Ne or $\mathrm{Ar}$ ) and expanded into the molecular beam source chamber (5.10-5 mbar during operation). The core of the resulting pulsed supersonic jet is extracted through a $2 \mathrm{~mm}$ diameter skimmer and enters the ion source of a $0.90 \mathrm{~m}$ long linear Wiley-McLaren type time-of-flight mass spectrometer (TOF-MS) that is or- 


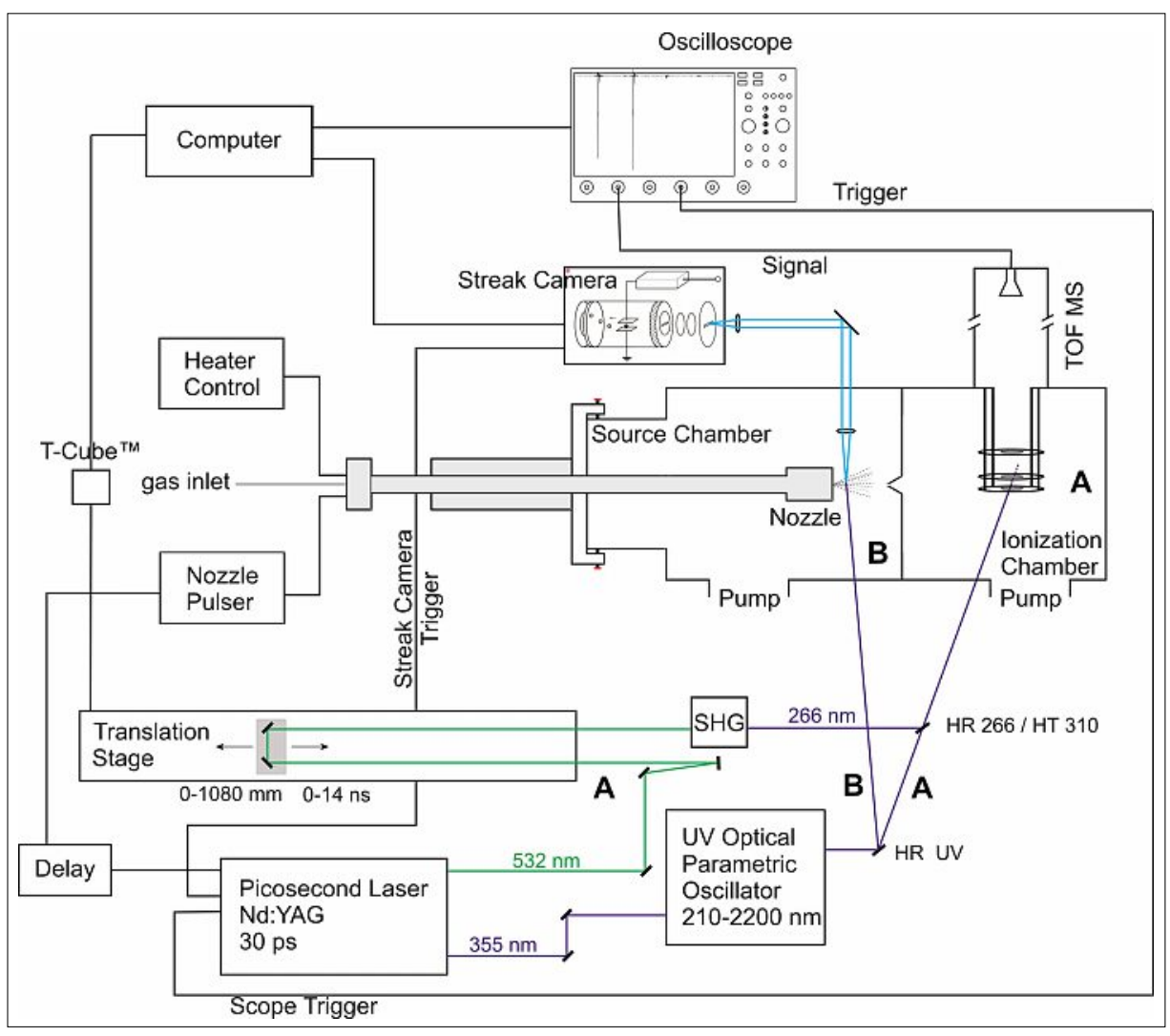

Fig. 1. Experimental scheme of the ps pump-ionization setup (A) and ps streak camera setup (B).

thogonal to the molecular and laser beams. The synchronization of the pulsed nozzle relative to the ps laser is controlled by a Berkeley Nucleonics Corporation (BNC) 575 digital delay/pulse generator.

A $20 \mathrm{~Hz}$ Nd:YAG ps laser system (EKSPLA PL2441, fiber oscillator in combination with a regenerative amplifier/ double pass amplifier) produces laser pulses of $\sim 25 \mathrm{ps} / 80 \mathrm{~mJ}$ at $1064 \mathrm{~nm}, 40 \mathrm{~mJ}$ at $532 \mathrm{~nm}$ and $24 \mathrm{~mJ}$ at $355 \mathrm{~nm}$. The $355 \mathrm{~nm}$ output pumps an EKSPLA PG401 UV optical parametric oscillator/amplifier (OPO/ OPA) system that is tunable from 210-350 $\mathrm{nm}$ and delivers $\sim 60 \mu \mathrm{J}$ pulses of $\sim 20 \mathrm{ps}$ length and $\sim 10 \mathrm{~cm}^{-1}$ bandwidth. The UV output is tuned to a molecule-specific frequency, collimated to a $3 \mathrm{~mm}$ diam. beam by two $\mathrm{f}=1000 \mathrm{~mm}$ lenses and crossed with the molecular beam inside the TOFMS ion source. The molecules are $S_{0} \rightarrow S_{1}$ excited by the UV OPO/OPA and then ionized by a $266 \mathrm{~nm}(\sim 20 \mathrm{ps})$ pulse that is generated by doubling the residual $532 \mathrm{~nm}$ with a Brewster-angle cut BBO. The time between pump and ionization pulses is varied by sending the latter over a $1080 \mathrm{~mm}$ long translation stage, resulting in 0-6 ns delays. The ionization pulse is spatially superimposed with the UV from the tunable OPO. The spindle of the translation stage is actuated with a DC micromotor driven by a Thorlabs T-Cube ${ }^{\mathrm{TM}}$ controller that is interfaced to a PC under LabView control. The exact travel distance is determined by a Faulhaber HEDS5500A optical encoder lens. The streak camera is triggered with a predelay (up to $-80 \mathrm{~ns}$ ) from the PL2441 Nd:YAG via a BNC 745 ps delay generator, allowing accurate $(<25 \mathrm{ps})$ and convenient (ps delay steps) pretriggering. The image at the streak camera output is recorded with a Hamamatsu ORCA-Flash 4.0 CCD. The shortest decay time that can be usefully measured is $\sim 30 \mathrm{ps}$. The Hamamatsu program saves the streak camera measurements as ITEX image files. These are first read in and converted into a data array. We employ a self-written time decay analysis in IDL using a multiexponential model and perform the fits with a LevenbergMarquardt routine.

\section{2-Aminopurine and its Water Clusters}

The ${ }^{1} \pi \pi^{*}$ state lifetime of bare 2 -aminopurine (2AP) has been previously determined from the Lorentzian broadening of the electronic origin $\left(0_{0}^{0}\right.$ band) rotational contour as $\tau=77 \pm 20$ ps. ${ }^{[13]}$ Nanosecond pump-probe measurements of the lifetime of $2 \mathrm{AP}$ and $2 \mathrm{AP} \cdot\left(\mathrm{H}_{2} \mathrm{O}\right)_{n} n=1$ and 2 clusters with the $\mathrm{H}_{2} \mathrm{O}$ located at the Sugar-edge site have indicated lifetimes $<3$ ns. ${ }^{[14,15]}$ Fig. 2 shows the pump-ionization decay traces of 2AP monomer and of the Sugar-edge water cluster isomers $I A$ and $2 A ;[14,15]$ the respective structures are included in Fig. 2 . The transients were measured at the re-

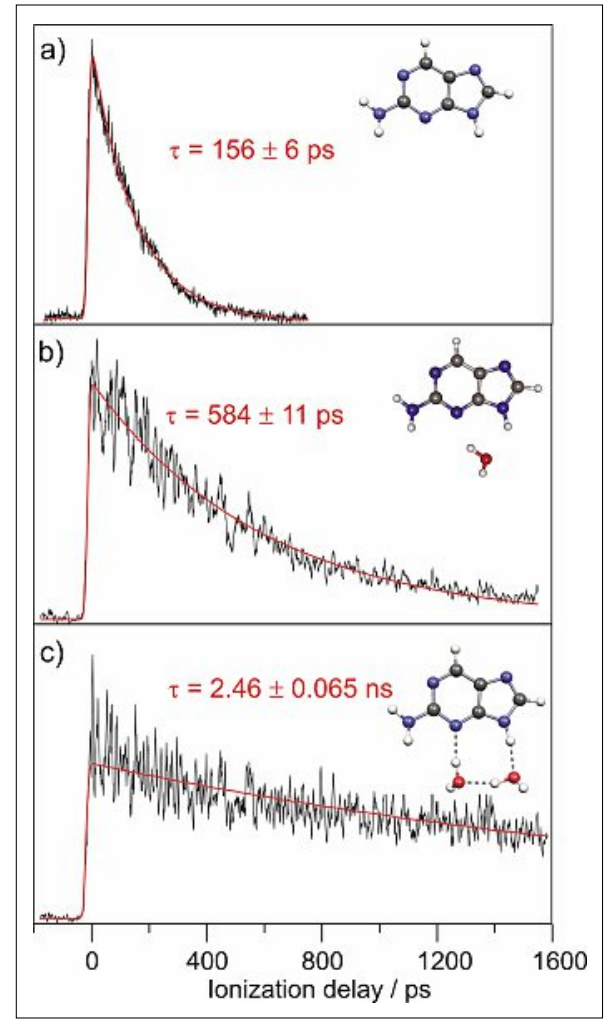

Fig. 2. Ionization delay transients of (a) 2-aminopurine, excitation at $32362 \mathrm{~cm}^{-1}$, (b) 2-aminopurine $\cdot \mathrm{H}_{2} \mathrm{O}$ isomer $1 \mathrm{~A}$, excitation at $32292 \mathrm{~cm}^{-1}$, (c) 2-aminopurine $\left(\mathrm{H}_{2} \mathrm{O}\right)_{2}$ isomer $2 A$, excitation at $32163 \mathrm{~cm}^{-1}$. 
spective $\mathrm{S} \rightarrow \mathrm{S}$ origins, which are 32362 $\mathrm{cm}^{-1}$ for 2AP, $32292 \mathrm{~cm}^{-1}$ for isomer $1 A$ of $2 \mathrm{AP} \cdot \mathrm{H}_{2} \mathrm{O}$ and $32163 \mathrm{~cm}^{-1}$ for isomer $2 A$ of 2AP. $\left(\mathrm{H}_{2} \mathrm{O}\right)_{2}{ }^{[14,15]}$ For $2 \mathrm{AP}$ we obtain a pump-ionization lifetime of $\tau=156 \pm 6$ ps. This is about twice the value derived from the rotational contour simulation. ${ }^{[13]}$ This indicates that while lifetime broadening has an effect on the rotational contour, there are additional broadening mechanisms that have not yet been taken into account. One of these is the inversion tunneling of the 2-amino group, which gives rise to two sub-bands and which may increase to the $0_{0}^{0}$ band width.

The lifetimes of the $2 \mathrm{AP} \cdot\left(\mathrm{H}_{2} \mathrm{O}\right)_{n} n=1$ and 2 clusters are $\tau=584 \pm 11$ ps for isomer $1 A$ and $\tau=2.460 \pm 0.065$ ns for isomer $2 A$. Hydrogen-bonding of one or two $\mathrm{H}_{2} \mathrm{O}$ molecules at the Sugar-edge site ( $1 A$, $2 A$ ) increases the $2 \mathrm{AP}$ lifetime by factors of 4 and 16 respectively. This reflects the influence of H-bonding on the lowest ${ }^{1} n \pi *$ excited state, which is near-degenerate with and closely coupled to the optically bright ${ }^{1} \pi \pi^{*}$ state. ${ }^{[16]}$ In bare $2 \mathrm{AP}$ this ${ }^{1} \mathrm{n} \pi^{*} /{ }^{1} \pi \pi^{*}$ coupling gives rise to rapid $(k=$ $\left.6 \cdot 10^{9} \mathrm{~s}^{-1}\right)$ nonradiative relaxation, which is slowed down by increasing solvation. ${ }^{[16]}$

These measurements reveal some advantages of the ps pump-ionization setup: i) The molecular and cluster lifetimes can be measured in a cluster mass-specific and often isomer-specific manner. ii) The time resolution - given by the temporal convolution of the pump and ionization pulses is $\sim 30 \mathrm{ps}$, which is typically $3-6$ times better than in the streak camera experiment, see below. iii) The photoions are collected and detected with near-unity efficiency. iv) Since several cluster masses are measured simultaneously, there is a potential multiplex advantage, and effects of cluster fragmentation can be diagnosed. Among the disadvantages, we note: i) Only one point on the pump/probe transient can be measured per shot. ii) Longer lifetimes than $\sim 3$ ns are increasingly difficult to measure because of the finite pointing stability of the delay stage and slow pointing drifts of the two lasers. iii) The density of molecules/ clusters is $\sim 500$ times lower in the TOFMS source than in front of the pulsed nozzle. iv) The ionization pulse energy cannot be increased without limits because of spurious one-color two-photon ionization processes.

\section{2-Pyridone Clusters}

We tested the ps streak camera setup using 2-pyridone (2PY) and its self-dimer (2PY), because their ${ }^{1} \pi \pi^{*}$ state fluorescence lifetimes have been previously determined via the Lorentzian widths of individual rovibronic lines as $\tau=11.4 \pm 0.1 \mathrm{~ns}$ for $2 \mathrm{PY}$ and $\tau=9.0 \pm 0.1 \mathrm{~ns}$ for $(2 \mathrm{PY})_{2} \cdot{ }^{[17,18]}$
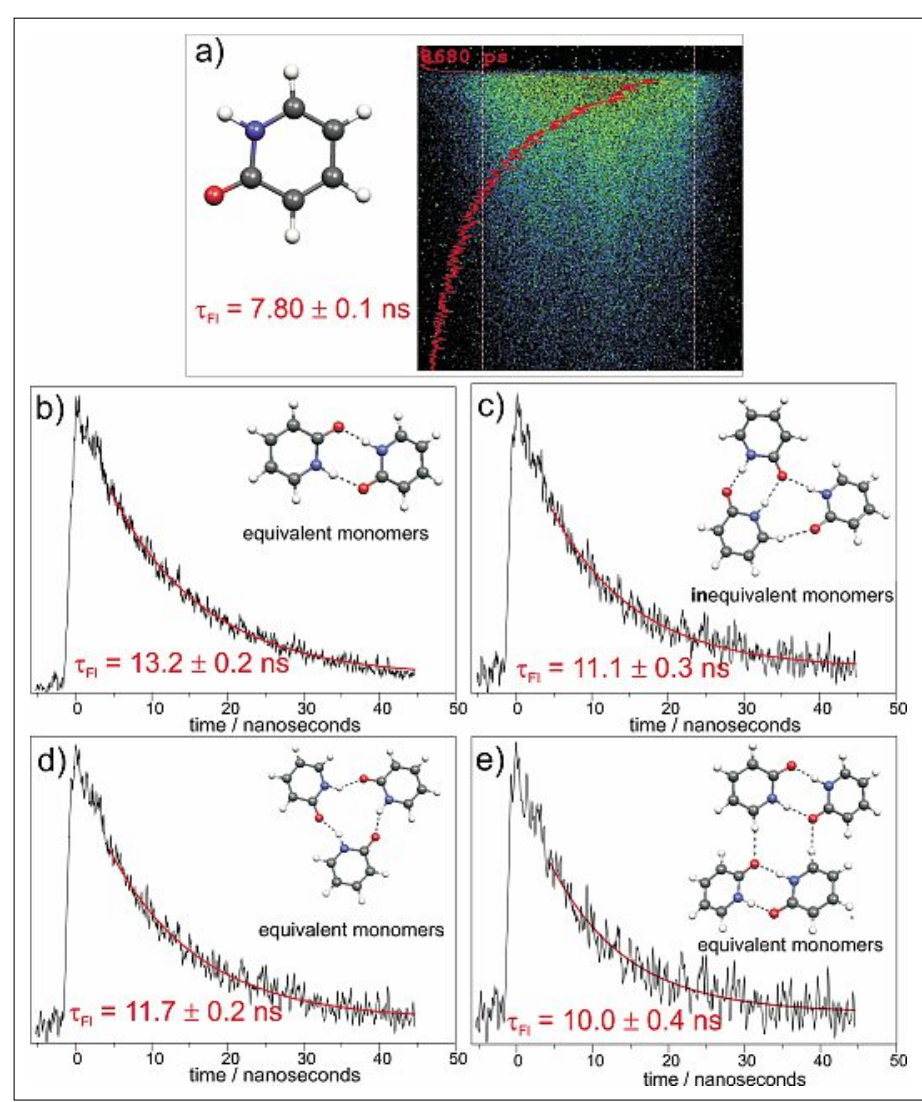

Fig. 3. Ps streak camera image of a) 2-pyridone fluorescence. Fluorescence time profiles and exponential decay fits of b) the (2PY) dimer, c) the $(2 \mathrm{PY})_{3}$ isomer $A$ d) the $(2 \mathrm{PY})_{3}$ isomer $B$ and e) the (2PY) tetramer.

Furthermore, we investigated two isomers of the 2-pyridone trimer $(2 \mathrm{PY})_{3}$, denoted $A$ and $B$, and the (2PY) homo-tetramer, which have been identified and assigned by resonant two-photon ionization spectroscopy. ${ }^{[19]}$ All species were excited at their respective $0_{0}^{0}$ transitions, which are at $29886 \mathrm{~cm}^{-1}$ (2PY), $30807 \mathrm{~cm}^{-1}$ (2PY), $30931 \mathrm{~cm}^{-1}$ for the low-symmetry (2PY) isomer $A, 30988 \mathrm{~cm}^{-1}$ for the high-symmetry (2PY) $)_{3}$ isomer $B$ and $30572 \mathrm{~cm}^{-1}$ for the $(2 \mathrm{PY})_{4}$ homo-tetramer. ${ }^{[19]}$ Fig. 3 shows the respective fluorescence lifetime measurements, lifetime fits and $a b$ initio optimized ground-state structures.

The ps streak camera lifetime of $2 \mathrm{PY}$ in Fig. 3 is fitted as $\tau_{\mathrm{fl}}=7.80 \pm 0.05 \mathrm{~ns}$ $(1 \sigma)$. This is somewhat shorter than the 11 $\pm 1 \mathrm{~ns}$ determined by Held et al. from the Lorentzian broadening of individual rotational lines. ${ }^{[17]}$ The lifetime of $(2 \mathrm{PY})_{2}$ is $\tau_{\mathrm{fl}}$ $=13.2 \pm 0.2 \mathrm{~ns}$. This $70 \%$ increase in lifetime relative to $2 \mathrm{PY}$ is in agreement with previous observations that bridging the $c i s$ amide $\mathrm{H}-\mathrm{N}-\mathrm{C}=\mathrm{O}$ group of $2 \mathrm{PY}$ with two or more $\mathrm{H}$-bonds prevents out-of-plane twisting of the $\mathrm{NH}$ and $\mathrm{C}=\mathrm{O}$ bonds in the ${ }^{1} \tau \tau^{*}$ state, thereby blocking the access to the lowest conical intersection of 2PY.[20] Held et al. determined the (2PY) ${ }_{2}$ lifetime via Lorentzian line-broadening as $9 \pm 1$ ns. ${ }^{[18]}$ Given that this lifetime is 4 ns shorter while the $2 \mathrm{PY}$ lifetime is $2.2 \mathrm{~ns}$ longer than the ps streak camera measurements, and given the much smaller fit errors of the latter, we suggest that the Lorentzian broadening lifetime determinations have a $\pm 2 \mathrm{~ns}(1 \sigma)$ uncertainty, which is twice that indicated in refs. [17] and [18]. In this case, the differences relative to the ps streak camera measurement lie within $\pm 2 \sigma$ for $2 \mathrm{PY}$ and $(2 \mathrm{PY})$

The lifetimes of the two isomers of $(2 \mathrm{PY})_{3}$ are intermediate between those of $2 \mathrm{PY}$ and (2PY) ${ }_{2}$. The $C_{3}$ symmetry isomer $B$ has a lifetime $\tau_{\mathrm{fl}}=11.7 \pm 0.2 \mathrm{~ns}$. The low-symmetry $C_{1}$ isomer $A$ has a slightly shorter lifetime $\tau_{\mathrm{fl}}=11.1 \pm 0.3 \mathrm{~ns}$. The lifetime of $(2 \mathrm{PY})_{4}$ was determined as $\tau_{\mathrm{fl}}=10.0$ $\pm 0.4 \mathrm{~ns}$. A reason for this pattern of lifetimes with increasing cluster size is that the $\mathrm{H}$-bonds are strongest in the dimer, which exhibits a planar $\mathrm{H}$-bonded 8-center ring. In the trimers and tetramer the H-bond arrangements are increasingly strained and twisted, ${ }^{[19]}$ which leads to a weaker clamping and allowing easier out-of-plane twisting of the $\mathrm{H}-\mathrm{N}-\mathrm{C}=\mathrm{O}$ group and more rapid radiationless relaxation. ${ }^{[20]}$

The ps streak camera setup has the advantage that: i) the local density in the beam is higher in front of the pulsed nozzle, ii) the entire lifetime range $(0.2-50 \mathrm{~ns})$ is measured for every laser shot, iii) the measurement accuracy for lifetimes $>500$ ps is good and becomes increasingly better with increasing lifetime. Disadvantages are: i) Fluorescence measurements are not intrinsically mass- or species-specific and may contain contributions from other fluorescent species. ii) The accuracy of lifetimes $<200$ ps decreases rapidly with decreasing lifetime. iii) The maximum efficiency for collecting photons is $\sim 3 \%$ and this cannot 
be increased without deteriorating the time resolution.

\section{Conclusions}

With the ps pump-ionization setup we have measured the excited-state decays of 2-aminopurine (2AP) and its water clusters $1 A$ and $2 A$ which could not be previously resolved with ns experiments. ${ }^{[13-15]}$ All fits resulted in monoexponential decays. The $\sim 155$ ps lifetime for the monomer is twice the value determined via Lorentzian line broadening of the 2AP rotational contours. ${ }^{[13]}$ This makes it clear that additional broadening mechanisms that were so far disregarded in the contour modeling must be taken into account. The lifetime of the 2AP chromophore is found to increase dramatically with the number of solvent water molecules, by a factor of four for the $1 \mathrm{~A}$ complex and by a factor of 16 for the $2 A$ cluster.
The fluorescence lifetimes of 2-pyridone (2PY) and of four different (2PY) clusters were determined with the ps streak camera setup. The 2PY monomer has the shortest fluorescence lifetime with $7.80 \mathrm{~ns}$, while the dimer has the longest lifetime of $13.2 \mathrm{~ns}$. The two trimers have a similar lifetime with $11.7 \mathrm{~ns}$ for isomer $B$ and $11.2 \mathrm{~ns}$ for isomer $A$. The fluorescence lifetime of the tetramer is even shorter with $10.0 \mathrm{~ns}$.

Received: January 17, 2014

[1] A. J. Campillo, V. H. Kollmann, S. L. Shapiro, Science 1976, 193, 227.

[2] A. Lauberau, J. Chem. Phys. 1975, 63, 2262.

[3] W. Kaiser, A. Laubereau, Chem. Phys. 1979, 6, 313.

[4] M. Iannone, B.R. Cowen, R. Diller, Applied Optics 1991, 30, 5247.

[5] R. M. Hochstrasser, D. S. King, A. C. Nelson, Chem. Phys. Lett. 1976, 42, 8.

[6] R. M. Hochstrasser, D. S. King, J. Am. Chem. Soc. 1975, 97, 4760 .
[7] P. M. Felker, W. R. Lambert, A. H. Zewail, $J$. Chem. Phys. 1982, 77, 1603.

[8] J. S. Baskin, A. H. Zewail, J. Chem. Phys. 1989, $93,570$.

[9] J. C. Williamson, J. M. Cao, H. Ihee, H. Frey, A. H. Zewail, Nature 1997, 386, 159.

[10] M. Kayano, T. Ebata, Y. Yamada, N. Mikami, J. Chem. Phys. 2004, 120, 7400.

[11] Y. Yamada, T. Ebata, M. Kayano, N. Mikami, J. Chem. Phys. 2004, 120, 7410.

[12] K. L. Reid, Int. Rev. Phys. Chem. 2008, 27, 607.

[13] S. Lobsiger, R. K. Sinha, M. Trachsel, S. Leutwyler, J. Chem. Phys. 2011, 134, 114307.

[14] R. K. Sinha, S. Lobsiger, S. Leutwyler, J. Phys. Chem. A 2012, 116, 1129.

[15] S. Lobsiger, R. K. Sinha, S. Leutwyler, J. Phys. Chem. B 2013, 117, 12410.

[16] S. Lobsiger, R. K. Sinha, S. Blaser, H.-M. Frey, S. Leutwyler, to be published

[17] A. Held, B. B. Champagne, D. W. Pratt, $J$. Chem. Phys. 1991, 95, 8732.

[18] A. Held, D. W. Pratt, J. Chem. Phys. 1992, 96, 4869.

[19] C. G. Heid, Diploma thesis, Dept. für Chemie und Biochemie, Universität Bern, 2008.

[20] S. Blaser, P. Ottiger, S. Lobsiger, H.-M. Frey, S. 